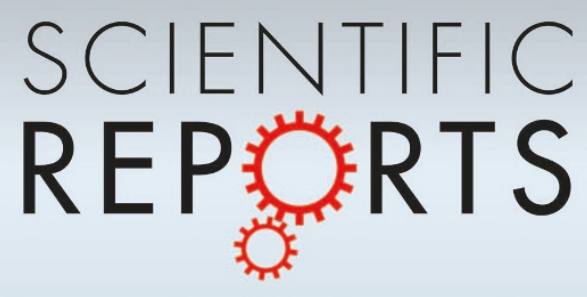

OPEN

SUBJECT AREAS:

ELECTRICAL AND

ELECTRONIC

ENGINEERING

MECHANICAL ENGINEERING

APPLIED PHYSICS

DESIGN, SYNTHESIS AND

PROCESSING

Received

20 November 2013

Accepted

5 March 2014

Published

4 April 2014

Correspondence and requests for materials should be addressed to J.L. (jliu@mail.ipc.ac.

cn)

\section{Personal electronics printing via tapping mode composite liquid metal ink delivery and adhesion mechanism}

\author{
Yi Zheng' , Zhi-Zhu He', Jun Yang' \& Jing Liu', ${ }^{1,2}$
}

\begin{abstract}
'Beijing Key Lab of CryoBiomedical Engineering and Key Lab of Cryogenics, Technical Institute of Physics and Chemistry, Chinese Academy of Sciences, Beijing 100190, China, ${ }^{2}$ Department of Biomedical Engineering, School of Medicine, Tsinghua University, Beijing 100084, China.
\end{abstract}

Printed electronics is becoming increasingly important in a variety of newly emerging areas. However, restricted to the rather limited conductive inks and available printing strategies, the current electronics manufacture is usually confined to industry level. Here, we show a highly cost-effective and entirely automatic printing way towards personal electronics making, through introducing a tapping-mode composite fluid delivery system. Fundamental mechanisms regarding the reliable printing, transfer and adhesion of the liquid metal inks on the substrate were disclosed through systematic theoretical interpretation and experimental measurements. With this liquid metal printer, a series of representative electronic patterns spanning from single wires to desired complex configurations such as integrated circuit (IC), printed-circuits-on-board (PCB), electronic paintings, or more do-it-yourself (DIY) devices, were demonstrated to be printed out with high precision in a moment. And the total machine cost already reached personally affordable price. This is hard to achieve by a conventional PCB technology which generally takes long time and is material, water and energy consuming, while the existing printed electronics is still far away from the real direct printing goal. The present work opens the way for large scale personal electronics manufacture and is expected to generate important value for the coming society.

\begin{abstract}
o date, printed electronics is incubating rather flourishingly in many industries owing to its superiority of unique flexibility, high production efficiency and low-cost over conventional electronics manufacture strategies. Clearly, the achieved technologies have increasingly contributed to modern electronics, especially in those newly emerging areas such as printable transistors ${ }^{1-3}$, flexible displays ${ }^{4}$, electrodes ${ }^{5,6}$, sensors ${ }^{7}$, antennas ${ }^{8}$, radio-frequency identification (RFID) tags ${ }^{9}$ and solar cells ${ }^{10}$ etc. So far, a variety of matched functional materials like conductive polymers ${ }^{11}$, nanoparticles ${ }^{12,13}$, carbon nanotubes ${ }^{14-16}$, inorganic semiconductors ${ }^{17}$ etc. have been tremendously investigated in order to quickly make out various desired electronic devices. And the fabrication strategies are enabled mainly by way of micro-contact printing ${ }^{18}$, screen printing ${ }^{19}$, roll-to-roll printing ${ }^{20}$ and inkjet printing ${ }^{21}$ etc. Meanwhile, it is also gradually realized that most of the currently available conductive inks still remained not ideal enough. One major tough issue lies in the yet discontent electrical conductivity of the available conductive materials. That is, most of such electronic inks possess much lower electrical conductivity than that of the metal materials, especially at room temperature situation ${ }^{22}$. Even some mitigation measures have been tried to acquire better conductivity, for example through high temperature post-treatment after patterning the inks on the substrates. Unfortunately, such endeavor would evidently increase the energy consumption and manufacturing cost. In addition, most of the flexible substrates generally request the post treatment temperature to be lower than $150^{\circ} \mathrm{C}$, which however will largely limit the electrical performance of the sintered materials. Further, many conventional printing technologies still suffer from other undesirable features such as complicated preparation process of conductive inks and relatively high cost thus involved. These factors restrain in a large extent their in-depth and ubiquitous adaptability in modern society, particularly for the personal use purpose.

Recently, a large class of room temperature liquid metal ink such as gallium (melting temperature $29.8^{\circ} \mathrm{C}$ ) and its alloy (melting temperature of eutectic gallium-indium $15.5^{\circ} \mathrm{C}$ ) increasingly attracted attentions over the world $^{23-27}$. The inborn nature of liquid and high conductivity of such metal fluid makes it a most promising candidate as electronics inks directly printable at room temperature. Aiming to establish a practical way for printing liquid metal inks, we have systematically evaluated the feasibility of a wide range of the existing printing strategies. However, it is unfortunate to find that many of the current methods failed to be useful for directly
\end{abstract}




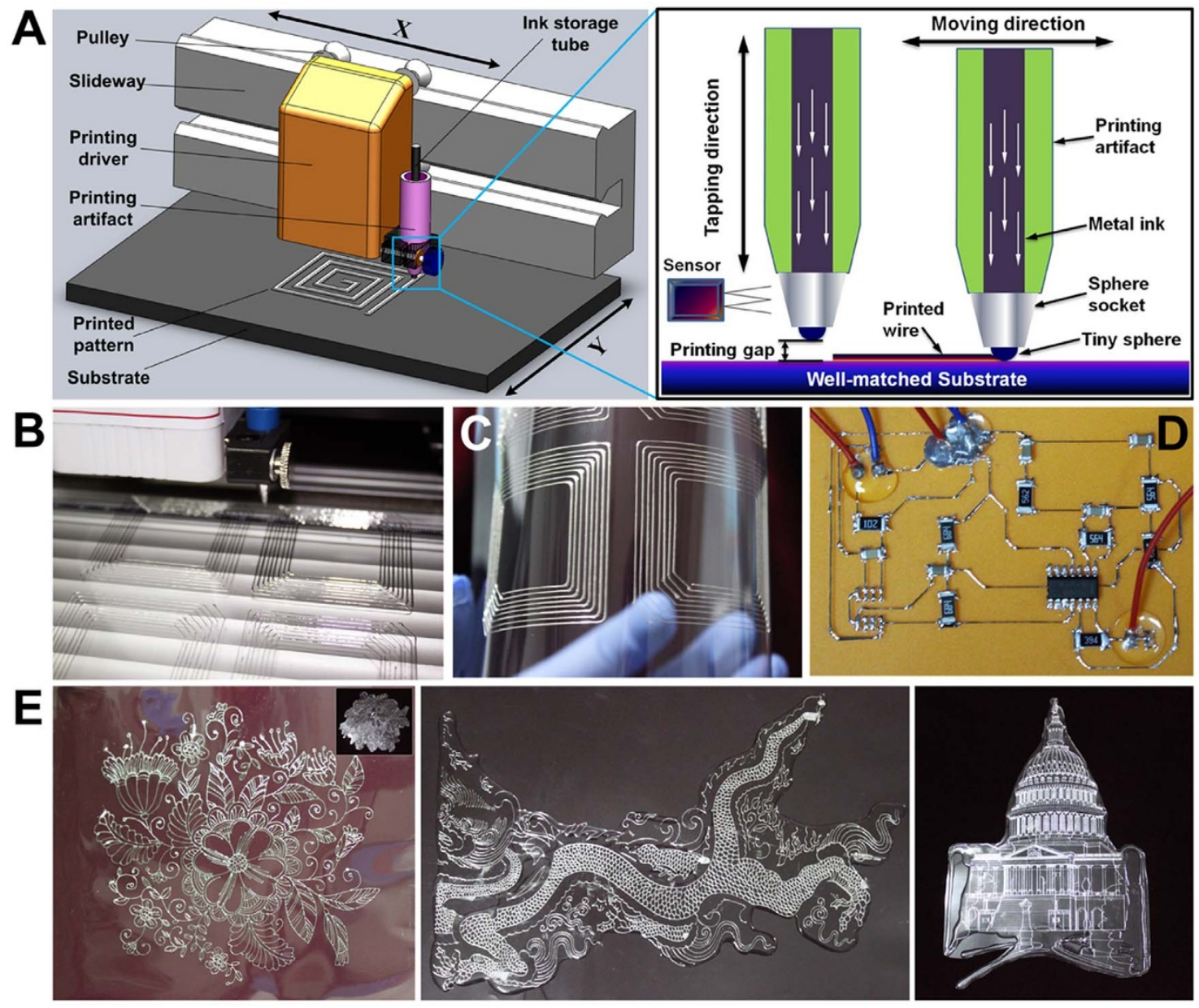

Figure 1 Schematic of the established liquid metal printer and the printed electronics pattern. (A), 3D diagram of the low-cost computer-controlled printer to illustrate the tapping mode movement of the printing head, the composite fluid mechanics to deliver, transfer and adhere the liquid metal ink to the substrate. (B), Physical photograph for the printing process of the machine. (C), Photograph of the RFID structure printed by liquid metal ink on flexible PVC film. (D), Printed circuit with IC chips incorporated; (E), Directly printed and packaged patterns as decoration drawings and building. (Jing Liu).

driving and printing out the liquid metal ink due to its extremely high surface tension $^{28-30}$. For example, dispensing method has been successfully applied to insoluble organic semiconducting materials and conductive silver ink $\mathrm{k}^{5,8}$. Theoretically, dispensing expels a continuous stream of material through the nozzle to the substrate, which is somewhat similar to inkjet printing. Its excellent feature lies in the ability to print inks with rather high viscosity. However, for liquid metal inks (such as GaIn24.5) with low viscosity and high surface tension, our practice indicates that the printed conductive lines through dispensing would sometimes spontaneously shrink to droplet which may seriously affect the quality of the final electronics pattern. The reasons can be attributed to that the liquid metal ink is sensitive to the pressure and speed of the dispensing, and this method could not sufficiently overcome the high surface tension effects. Meanwhile, another major printing method, the laser sintering is mainly applied on metals with high melting point. For this case, the high temperature generated from the laser would easily destroy the PVC flexible substrate. Besides, the laser sintering will increase both the energy-consumption rate and the cost. Therefore, it is also not in line with our low-cost objective. In order to break up the bottleneck seriously impeding the wide range applications of the printed electronics, here we present an innovative solution to address the urgent issues lying behind, by foremost developing a tappingmode enabled composite fluid delivery mechanism and adopting the room-temperature liquid metal as electronic inks to directly and automatically print out target electronics.
With this landmark liquid metal printer, a series of desired electronically conductive patterns spanning from single wires to various complex structures like integrated circuit (IC), antenna, PCB, RFID, electronic greeting cards or paintings, decoration arts, classical building schemes (White House, Great Wall etc) or more do-it-yourself (DIY) circuits and devices were demonstrated to be printed out with high precision in a moment. The electronic performance of the printed device has also realized the desired target. Particularly, the total cost for the machine already reached rather low price which should be personally affordable. This is hard to achieve otherwise by the latest advanced technologies since conventional PCB making generally takes long time and is material, water and energy consuming while the existing printed electronics is still far away from the real direct printing purpose. As a straightforward and evidently "beyondthe-lab" technology, the present work opens the way for home level electronics making which is expected to generate important value for the society's coming daily life.

\section{Results}

Personal liquid metal printer and basic working features. In this study, our developed computer-controlled liquid metal printer (Fig. 1A) is mainly composed of an automatic printing driver, an artifact ink cartridge, two pulleys and a notebook computer preloaded with controlling software. The driver with built-in monitoring sensor is capable of duly realizing tapping motion of the cartridge to facilitate delivery of the liquid metal ink and set 


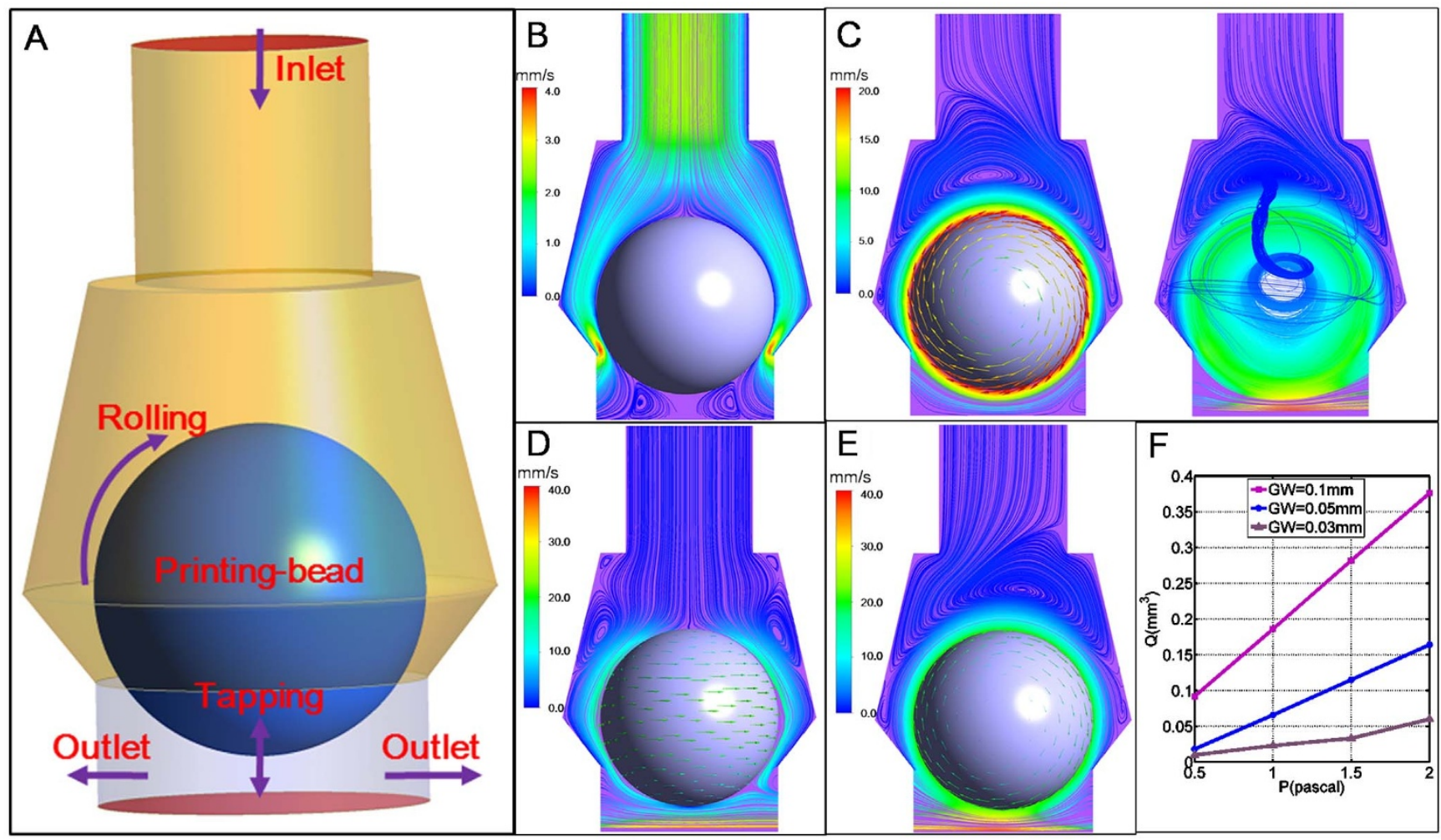

Figure $2 \mid$ Composite fluid mechanics for delivering, transferring and printing the liquid metal inks. (A), Illustration for liquid metal delivery through the small gap between roller-bead and its surrounding seat; The flow streamline of the liquid metal ink in the gap for the conditions: (B), The driven pressure from the inlet is used for overcoming the flow resistance in the socket, $\mathrm{P}_{\text {in }}-\mathrm{P}_{\text {out }}=1 \mathrm{~Pa}, \mathrm{~V}=0$ and $\omega=0$; (C), The rotation of the roller-bead can enhance the ink outflow through the gap, $\mathrm{P}_{\text {in }}-\mathrm{P}_{\text {out }}=1 \mathrm{~Pa}, \mathrm{~V}=0$ and $\omega=60 \mathrm{rad} / \mathrm{s}$ along the $\mathrm{y}$ axis; $(\mathrm{D})$, When roller-bead rotating along the $\mathrm{z}$ axis and completely sliding on the substrate, it has no contribution to the outflow of the ink and induces the printing failure, $\mathrm{P}_{\text {in }}-\mathrm{P}_{\text {out }}=1 \mathrm{~Pa}, \mathrm{~V}=0.004 \mathrm{~m} / \mathrm{s}$ and $\omega$ $=60 \mathrm{rad} / \mathrm{s}$ along the $\mathrm{z}$ axis; (E), A stable composite fluid transfer state plays a key role in the high-quality printing, $\mathrm{P}_{\text {in }}-\mathrm{P}_{\text {out }}=1 \mathrm{~Pa}, \mathrm{~V}=0.004 \mathrm{~m} / \mathrm{s}$ and $\omega$ $=60 \mathrm{rad} / \mathrm{s}$ along the y axis. (F), The outflow flux of the liquid metal ink for different driving pressures (P) and gap widths (GW). (More detailed explanations on the parameters are available in our supplementary materials) (Jing Liu).

aside a printing gap to avoid destroying the pre-patterned electronics. When one wishes to print the selected vector graph, the printer driver loaded with the developed ink cartridge would move under software guidance along the $\mathrm{X}$ direction. Meanwhile, the substrate moves along the $\mathrm{Y}$ direction harmonically to accomplish the electronics manufacture task. Fig. $1 \mathrm{~B}$ presents a schematic picture of the printing process and Fig. 1C shows the photograph of the printed RFID made of liquid metal ink with excellent quality. Fig. 1D illustrates a practical device which has combined the currently printed circuit with several necessary IC chips. For making a more complex electronics pattern as desired, the present printer also works rather reliably. What presented in Fig. $1 \mathrm{E}$ are electronically conductive structures to represent decorative drawings, or classical building schemes. Clearly, the uniformity, smallest line width etc. of the patterns has already reached a very high precision like $100 \mu \mathrm{m}$, compared with the most advanced direct writing electronics. Readers can find such resolution details and related influencing factors in later sections. Meanwhile, a short movie for illustrating the basic working principle of the liquid metal printer and practical printings of a variety of conductive patterns was also made for the readers to better understand more specific details of the current technology.

Composite fluid delivering mechanism to transfer and print liquid metal ink. The mechanically controlled ink rolling-transfer and printing mechanism is a core to guarantee the reliable running of the current high performance liquid metal printer. The roller-bead (700 $\mu \mathrm{m}$ in size) in the socket (Fig. 2A) is enforced from pressure of the liquid metal ink to tightly fit with the gap to prevent outflow of the ink during the non-operating state (Fig. 2B, C). As printing being started-up through notebook computer, the printing head is driven to move at a specific direction and velocity. Meanwhile, the rollerbead taps to the substrate and the gap is then opened to allow ink outflow with the assistance of roller-bead rotation. Under pressure from the driver, the liquid metal ink with high density are brought out along with the rolling bead and then transferred to the wellmatched substrate. The rhythmic tapping motion of the rollerbead brings along the oscillation of roller-bead at the gap to remove impurity and maintain smooth flow. The fluid dynamics behavior of the liquid metal in the working state is presented in Fig. 2 (B-E), where the simulated flow streamlines of the liquid metal inks under different pressure and speed conditions are illustrated (more detailed analysis is available in our supplementary material). To evaluate the impact of the gap width induced by the printing head's tapping motion on the outflow of the ink, additional simulations were also performed. Figure $2 \mathrm{~F}$ presents the outflow flux of the liquid metal ink through the gap for different driving pressures and gap widths. Clearly, the gap width plays a critical role in determining the flow flux of the ink. When the gap width tends to zero, the liquid metal ink stops outflow. Overall, the smooth outflow of the ink through the gap is dominated by the driving pressure, the rotation of the roller-bead, and the gap width which all were self-adjusted by the tapping motion. The core of this fundamental printing mechanism for the liquid metal printer can be classified as five key steps, i.e.: 1. Optical sensor to detect the distance between the moving bead and the printing substrate so as to precisely control the tapping motion of the printing head through computer feedback mechanism; 2. Planar mechanical movement of the 

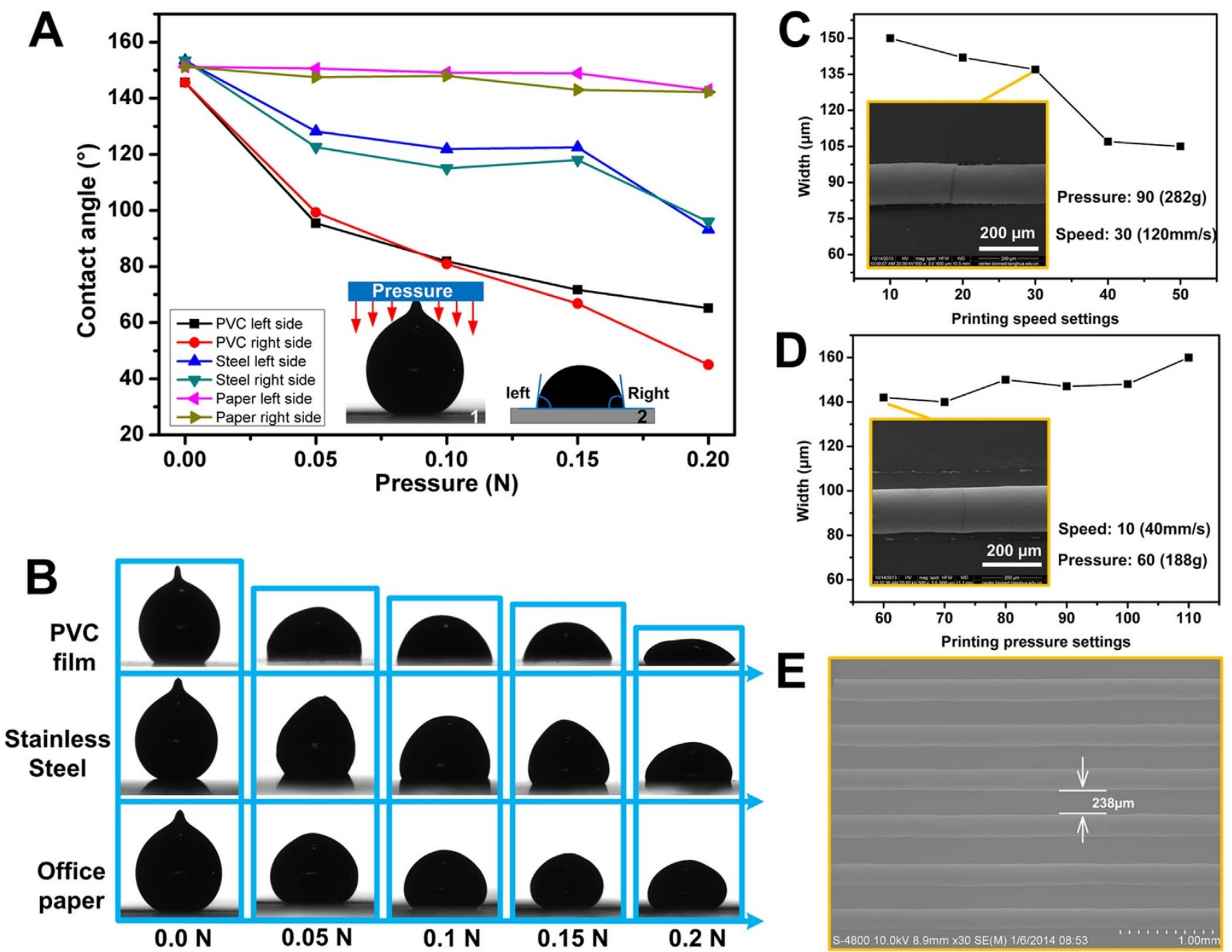

Figure 3 Effects of external factors to affect the acquired contact angle of GaIn24.5 droplet and the printing resolution. (A), The measured contact angles of GaIn24.5 droplet on PVC plastic film, stainless steel, office paper, respectively, as a function of impressed external minute-pressures increasing from 0 to $0.2 \mathrm{~N}$. The inset pictures present the schematic of the impressed pressure from upside (1) and illustration of the contact angle in both sides (2) which may be different resulting from a lopsided impressed pressure. (B), The wetting effect of the liquid metal droplets on the above-mentioned three kinds of substrate under ascendingly impressed external pressure, exhibiting that GaIn24.5 droplet owns excellent wettability on the PVC thin film. (C), The widths of the printed liquid metal track under constant pressure (set as $90^{\circ}$ ) and increasing printing speed, The inset picture shows the SEM of the printed liquid metal circuit at a speed of $30(120 \mathrm{~mm} / \mathrm{s})$. (D), Widths of the printed liquid metal track under constant speed (set as 10) and increasing printing pressure. The inset picture gives the SEM image of the printed liquid metal circuit at a speed of 30 (120 mm/s). (E), Distance between two printed adjacent conductive lines, about $200 \mu \mathrm{m}$. (Jing Liu).

printing head to guarantee a highly stable rolling of the roller-bead during printing and reducing its sliding activity (Fig. 2C, D); 3. Tapping and gravity enabled delivery of liquid metal to the tip bottom (Fig. 2B); 4. Automatically justified smooth flow of liquid metal through the gap between the roller-bead and its surrounding seat (Fig. 2E); and 5. The self-adapted balance between surface tension of the liquid metal and surface energy of the substrate (restricting the stable shape of the liquid metal line) guarantees the reliable working of the printing machine. It is also quite noteworthy that the wettability of the liquid metal ink on the printing substrate must be better than the stainless steel printing bead. Only in this way can the inks be smoothly dropped off from the roller-bead surface and then adhered to the substrate.

Wetting properties between liquid metal ink and matched printing substrate. The wettability of $\mathrm{GaIn}_{24.5}$ ink on the target substrate is also a key factor affecting the printing quality. Several "paper like" materials were comparatively tested to identify a well-matched printing material. So far, the flexible PVC thin-film exhibits the best outcome. To further clarify the mechanisms, the contact angles of $\mathrm{GaIn}_{24.5}$ ink on universal office paper, "paper like" flexible transparent polyvinyl chloride (PVC) thin film, stainless steel were measured, respectively, in order to quantitatively evaluate their compatibility with $\mathrm{GaIn}_{24.5}$ ink. The sitting drop method was applied to form the liquid metal micro drop and five points fitting method was adopted to compute all the acquired contact angles. Afterwards, increasing external minute-pressure was clamped down to evaluate the change of the contact angles. Fig. 3A exhibits the measured results of the liquid metal ink dropped on the above-mentioned three materials under diverse exerted pressures. It can be obviously discovered that when there is no external force, $\mathrm{GaIn}_{24.5}$ ink actually shows apparent non-wetting phenomenon on all surfaces $\left(\theta>90^{\circ} \mathrm{C}\right)$, which is considered to be mainly resulted from the large surface tension of the liquid $\mathrm{GaIn}_{24.5}$ alloy. Secondly, the applied pressure is able to facilitate wetting of $\mathrm{GaIn}_{24.5}$ ink on all substrate materials in varying degrees. 
Simultaneously, after exerting tiny pressure from upside, the PVC film substrate appears to have the best sensitivity to the impressed pressure. With the increase of the external pressure, the contact angle decreases sharply. However, the office paper case just decreases slightly under the same external pressure, indicating that the GaIn $_{24.5}$ ink owns much better wetting capability on PVC substrate than office paper, especially under large external pressure. Further, it can be noticed that there is certain difference between contact angles of the liquid metal on two sides of the PVC substrate. This was attributed to the effects caused by the slightly unevenly applied pressure and the favorable compatibility between liquid metal ink and the PVC substrate. It is easily accepted that the better compatibility, the more prone for the liquid metal ink to result in different contact angles under the same uneven force. When implementing a pressure of $0.1 \mathrm{~N}$, the $\mathrm{GaIn}_{24.5}$ ink becomes to exhibit wetting with the PVC film $\left(\theta<90^{\circ}\right)$. When increasing to $0.2 \mathrm{~N}, \mathrm{GaIn}_{24.5}$ ink displays excellent compatibility with the PVC film. Therefore, there is a completion described as follows: Wettability $_{\text {pvc film }}>$ Wettability $_{\text {steel }}>$ Wettability $_{\text {office paper. }}$

In accordance with the above-mentioned transfer printing mechanism, the PVC thin film was really a kind of well-matched printing substrate for the presently developed technique. When performing the printing task, the pressure generated from the printing driver subtly precipitates the effluent metal ink to be well printed on the matched PVC film.

At this stage, diverse flexible electronics have been able to be quickly printed out with high precision through the present system. This provides an extremely easy going way to make electrical devices which is hard to do otherwise via conventional strategies. The computer-controlled automatic printing allows users to easily make any desired 2D conductive patterns "beyond the lab", just by inputting the requested images in an appropriate vector format. Practically, the printer is able to function at a printing speed of $0 \sim 400 \mathrm{~mm} / \mathrm{s}$ (preferred $0 \sim 200 \mathrm{~mm} / \mathrm{s}$ to ensure the print quality). And a printing pressure in the range of $0 \sim 800 \mathrm{~g}$ was available. Along this way, the resolution of the printed circuits was measured through a scanning electron microscope under various printing speed and percussion pressure, which are both considered to affect the quality. The width values acquired under varied speeds and pressures are presented in Fig. 3C and D, respectively. Fig. 3C shows the widths of the printed liquid metal track under constant pressure (set as $90^{\circ}$ ) and increasing printing speed. Clearly, as the speed increases, the printed line width becomes gradually smaller. The inset picture shows the SEM image of the printed liquid metal circuit at a speed of $30(120 \mathrm{~mm} / \mathrm{s})$. Fig. 3D exhibits the widths of the printed liquid metal track under constant speed (set as 10) and increasing printing pressure. There is a slight increase in the width with the ascending of printing pressure. The inset shows the SEM picture of liquid metal wire at a pressure of 60 (188 g), revealing excellent stability and uniformity.

Meanwhile, it can also be noticed that the slower the speed, the more stable and uniform the lines. When set the printing speed to an over large value, the printed outcome would be completely dissatisfied. Therefore, the printing speed affects more on the quality. In this case, an available printing speed is preferred to be set in the range of $0 \sim 50(0 \sim 200 \mathrm{~mm} / \mathrm{s})$, which can efficiently meet the acquirement in electronic fabrication. In addition, the presently developed method for printing liquid metal inks owns a wire resolution of $100 \mu \mathrm{m}$, which is a pretty high-quality printing among the currently available methods ${ }^{31}$. The minimum distance between two adjacent conductive lines is mainly determined by the liquid metal and the minimum moving step of the printing bead. Further, due to the large surface tension of the liquid metal alloy, there should be a minimum distance between conductive tracks to ensure the good function. In this way, a scanning electron microscopy measurement was carried out to show the spatial resolution of the developed method. The acquired SEM image of conductive line array exhibits an acceptable distance of $238 \mu \mathrm{m}$ between two adjacent conductive lines (as presented in Fig. 3E). This spatial resolution of the metal line was considered to be greatly dependent on the printing precision of the facility. In the near future, the printed liquid metal line with better resolution (even nanoscale) can still be possible given further promotion. Regarding the working reliability of the printed liquid metal circuits, appropriate methods were also established in the present study. According to former works, $\mathrm{PDMS}^{26}$ and room temperature vulcanization (RTV) silicone rubber $^{30}$ have been found capable of serving as the flexible packaging materials to ensure the environmental and mechanical stability of the circuit, without affecting its electrical performance. Some of such packaged electronics can be found in Fig. 1E and our supplementary materials.

Demonstration experiments on personal electronics manufacture. So far, the prototype machine of the present liquid metal printer as established in this work has been able to print out various electrically conductive patterns spanning from spots, lines, curves, to complex structures. The whole process is entirely automatic and completely controllable through a notebook computer. Basically, the printing of the target circuits depends on its mathematical expressions regarding geometrical domain, which means that the originally printable drawing should be vector graph. Therefore, before printing, the initial electronic draft should subject to an evaluation and necessary transformation between different figure formats. Regarding the performance, the currently achieved high quality printing is already practical enough to fulfill many practical needs in electronics design and manufacture.

To demonstrate the diverse capability of the liquid metal printer in manufacturing various electronics drafts, here we choose to print several most typical metal elements which can be used to compose complex electrical circuits or devices. In addition, a few potential areas where personal electronics manufacturing is expected to play important roles were also illustrated through interpreting their potential functions and the fundamental issues lying behind. The order to present the printed conductive targets starts from simple electronic elements to complex patterns until finally functional electronics. As is fully demonstrated, nearly any desired electronically conductive patterns can be directly printed out with high quality through the explored computer-controlled liquid metal printer.

In electrical engineering, a circuit is generally composed of various basic electrical elements such as straight line, polyline, circle or complex curve. With careful pre-design and selection of appropriate liquid metal ink, an electrical circuit with desired features such as size, structure, resistance etc. can be automatically printed out. Figure 4 presented several printed geometrical primitives. Clearly, with a design draft in mind, the corresponding electronic wires or signs (Fig. 4A, C) can be quickly printed out. The present machine also works well for printing characters either in English or Chinese (Fig. 4B). What printed in Fig. 4C are several 2-D conductive patterns. From the perspective of circuit, the printed close structure owns the same potential, which makes the routing manufacture of complex circuit in office rather efficient. Except for making the circuits, the present method also offers the tool for quick production of a series of high-performance electronic sensing elements which is significant for large-scale application of many functional electronic devices. For example, a direct printing of interdigitated-array microelectrodes (IDAM) and flexible antenna by the current printer is also demonstrated here, which is rather useful for making a group of detection sensors. Such an antenna composed of conductive line made from liquid metal ink is presented in Fig. 4D. What one needs to do for the printing is only the antenna size such as grid space $(4.8 \mathrm{~mm})$ and numbers $(7 \times 15)$ which is to determine the resonance frequency of the antenna. Once thing needs to mention is that, the electrical characteristics of the printed circuit are essentially related to the conductor and the substrate materials. The printed circuit has 

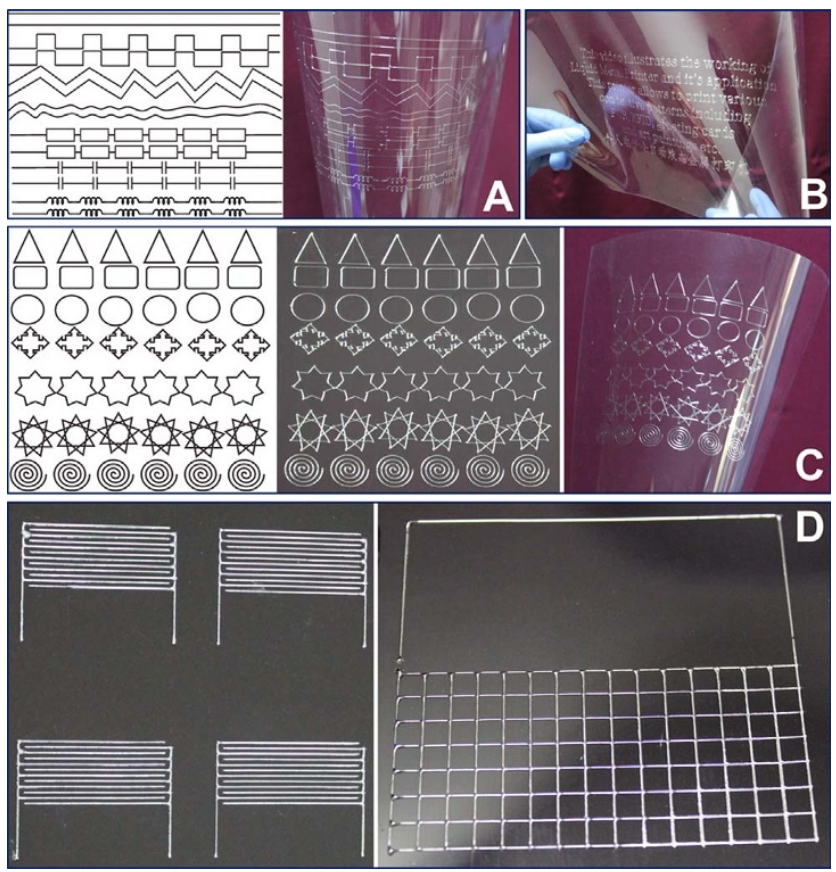

Figure $4 \mid$ Printed fundamental elements of lines, curves or characters to compose electronic circuits or devices. (A) Original geometrical design drafts for lines and wires vector graphs and printed results. (B) Printed conductive characters in English and Chinese. (C) Pattern vector graph, printed flexible object and its folding state. (D) Quick production of highperformance electronic elements such as interdigitated-array microelectrodes or radiofrequency identification antenna. (Jing Liu).

very small dissipated power due to low electrical resistivity of the liquid metal inks. In addition, substrate materials considered here have very low permittivity values and dissipation factors, which are desired for high-speed, high-frequency printed circuits. On the other hand, the electrical stability under mechanical stress is also a crucial aspect for the flexible conductive tracks. According to our former related researches ${ }^{30,32}$, the electrical resistance of the well-packaged liquid metal on PVC substrate was measured by digital electric bridge under varied bending angles. And the result revealed that the resistance there just shows a slight change under different bending angles, which is considered to be mainly caused by the effect of contact resistance. Therefore, the outcome confirms that the packaged liquid metal lines possess excellent stability even subjecting to mechanical bending.

Clearly, the present electronics printing method can be applied for manufacturing more other complex electrical devices with additional functions based on the basic concepts enabled herein. In fact, this principle has generalized purpose and can be extended to many different areas even daily life. An expected exciting future would be that people even children without particular training or experiences on electronics can easily print out their own functional devices in the coming time. The only requirement is just to download electronic drafts, print them out and then assembly all the items together as an integrated device.

Figure 5 exhibits several printed functional patterns made of liquid metal ink. Figure 5A and C show two printed integrated circuits (IC) on transparent PVC film with favorable quality, whose printing took only about 10 minutes. The excellent flexibility of the printed IC is presented in Fig. 5B, which can be adopted for making ending electronic devices. This highly efficient electronics fabricating strategy consumes no additional energy except for the power to drive the printing cartridge and therefore completely falls in the category of "green manufacture". Fig. 5D exhibits a printed Christmas tree accompanying with the beneath greeting words "Merry Christmas!"
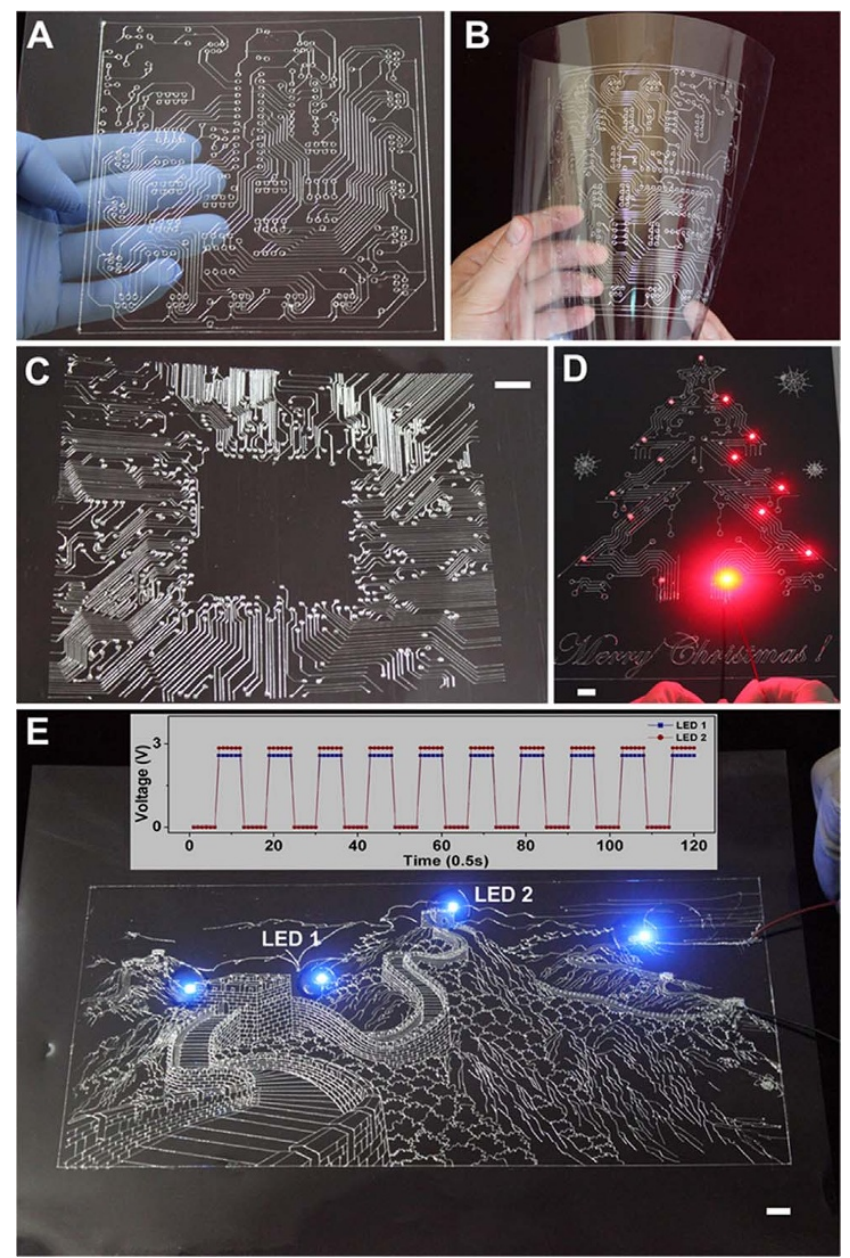

Figure 5 The quickly printed out functional electronic patterns via liquid alloy printer. (A), Physical picture of a printed-circuits-on-board for IC application. (B), Folding state of the PCB. (C), Another different printed integrated circuits on PVC substrate. (D), Printed electronic "Christmas tree" composed of liquid metal wires and English characters "Merry Christmas!" which is illuminated by several posting-type LEDs. (E), Manufactured lighting on liquid metal "Great Wall" through the developed method. (All the scale bars are $10 \mathrm{~mm}$ ) (Jing Liu).

which are composed of liquid metal wires illuminated by a few posting-type LEDs, showing the practicability of the printed circuits. Further, a more sophisticated electronics drawing manufacture, electronic painting of the world-famous "Great Wall", is printed on the flexible PVC thin-film and chromatically lighted up by LEDs (Fig. 5E). It indicates that the personal electronic devices can now be easily printed out in a moment. The liquid metal line exposed to the air will rapidly form a thin oxide skin to maintain the shape in a degree. The insert picture in Fig. 5E depicts the transient voltages of the two LED lights (1 and 2) with the splashing which shows very stable working property of the printed circuits. Therefore, reliable devices and complicated liquid metal patterns are all able to be manufactured through this straightforward and highly efficient composite printing way. Overall, the present method has generalized purpose in making various consumers oriented electronic patterns. More extensive applications can be found in our supplementary materials.

\section{Discussion}

As is increasingly realized, the liquid metal could serve as a perfect electronic ink for directly writing out electrical device. However, a big bottleneck in impeding the successful printing of such solutions lies in its extremely high surface tension. Through a series of comparative 
experiments, we found that the liquid metal is hard to be delivered smoothly inside the cartridge and then deposited on the target substrate via the conventional printing methods such as ink-jet or dispensing printing. As an alternative to those classical principles, which strongly depend on the driving pressure directly applied on the ink, the current liquid metal printing is entirely made possible through the tapping mode enabled composite fluid transfer mechanism which is consisted of a series of complex parallel actions of the printing head.

During the electronics manufacture, the tapping mode movement of the printing head is the core of the method. When the printinghead maintains at stationary state, the static roller-bead is enforced to locate at the bottom of its surrounding seat due to pressure of the liquid metal, which leads the roller-bead to tightly fit the gap to prevent outflow of the ink. However, the roller-bead rhythmically taps to the substrate during the working state so that the gap is opened continuously to allow ink outflow with the assistance of roller-bead rotation. The rhythmic tapping motion induces the oscillation of the roller-bead around the gap to remove impurity and maintain smooth flow, which is critical for the long time printing. Compared with the conventional printing method (such as microinject-printing), the tapping mode of the roller-bead owns the function of self-clearance such that it adapts excellently to print the liquid metal ink. In addition, the opening amplitude of the gap can be automatically adjusted through continuous tapping motion to uniformly control the ink outflow. When the printed electronic patterns are exposed to the air, the liquid metal ink $\left(\mathrm{GaIn}_{24.5}\right)$ quickly forms a thin layer (about $1 \mathrm{~nm})^{27}$ of gallium oxide on its surface, which prevents further oxygen transport from the air and avoid subsequent oxidation. When soldering with other electronics elements (such as resistor), their contact points are immersed within the liquid metal, which cannot be affected by the thin oxide skin of the liquid metal surface. A fast packaging has also contributed to maintain the reliability of the complex printed electronic patterns.

Compared with the existing printing strategies, the current method for printing electronics requests much less pre-processing and post-processing procedures. It also easily reached the applied metal resistivity. Further, our developed method for printing liquid metal inks already realized a very high wire resolution of $100 \mu \mathrm{m}$. And to ensure the quality of the manufactured conductive track, the printing speed is preferred to be set in the range of $2.4-12 \mathrm{~m} / \mathrm{min}$, which is among the quickest way in the high-quality printing meth$\mathrm{ods}^{31}$. In summary, we have demonstrated a pervasive way to automatically print out diverse electronic patterns on transparent plastic film via liquid metal printer. This composite fluid delivery mechanism for printing liquid metal ink is rather convenient, efficient and most important of all, extremely low-priced, implying its huge potentials to be widely adopted in smart electronics fabrication over the world. Its unique virtue is especially important in the increasingly emerging areas of personalized consumer electronics, with the capability of freely making various PCB, integrated circuits (IC), and functional electronics, via the do-it-yourself (DIY) style. The electrical characteristics of the printed electronics achieved the high quality and reliability expectations. In the near future, making little effort and under auxiliary of additional inks made from oxide, nanoparticle, semiconductor and carbon nano-structure, the present tapping mode composite ink delivery and printing mechanism can still be extended to more optoelectronic manufacture areas such as printing flexible display, transistors, solar cell, paper batteries and other multifunctional devices. This easily controllable and personally affordable system opens the way for large scale application of the printed electronics and offers a powerful office tool for electronics manufacture in the coming daily life.

\section{Methods}

Preparation of liquid metal electronic ink and matching printing substrate. As a remarkable material with favorable metallic conductivity, flowability, direct printability, and affordable cost, the EGaIn ${ }_{24.5}$ alloy was introduced here as the electronics ink which owns a melting point of $15.5^{\circ} \mathrm{C}$ and is therefore capable of remaining in liquid phase under room temperature. Further, its large density $\left(6280 \mathrm{~kg} / \mathrm{m}^{3}\right)$ and low viscosity $\left(2.7 \times 10^{-7} \mathrm{~m}^{2} / \mathrm{s}\right)$ make $\mathrm{GaIn}_{24.5}$ a preferable electronic ink. Such room temperature liquid metals have recently attracted big interests and were preliminarily utilized to fabricate simple and reliable flexible devices $^{23-27}$

According to the experiments, oxide in the alloy can significantly contribute to the adhesion but immensely reduce the liquidity. To maintain the favorable fluidity of the liquid metal alloy, herein, the adopted $\mathrm{EGaIn}_{24.5}$ alloy was not deliberately oxidized. For making the electronic ink, high-purity gallium and indium metals (with purity of 99.99 percent) were weighted as source materials with a ratio of $75.5: 24.5$ in line with the chemical compositions. Then the weighted gallium and indium metals are mixed together in the beaker which was beforehand cleaned by the deionized water and heated to $50^{\circ} \mathrm{C}$ until metals were fused completely, then stirred slightly. Afterwards, the configured $\mathrm{GaIn}_{24.5}$ ink with traces of natural oxide was injected into the ultrasonic cleaned core of the printing head.

Meanwhile, flexible materials suitable as "typical printing substrate" such as commercially available office paper, flexible PVC thin-film were both tried to screen out well-matched printing substrate. A contact angle meter (JC2000D3, Shanghai, China) was used to measure the contact angle of $\mathrm{GaIn}_{24.5}$ on different substrates under various external minute-pressures. Through a comprehensive evaluation, the PVC thin film with both transparent and flexible features was particularly identified as a perfect printing substrate in the present work.

Set up of the liquid metal printing platform. According to a series of our comparative experiments, the liquid metal inks as fabricated above are extremely hard to be driven by most of the currently available printing technologies such as direct writing, fluid dispensing, and micro-contact printing etc. For example, for the directprinting ways such as thermal bubble and piezoelectric inkjet printings, the liquid metal ink could not be driven so far, due to its either high vaporization temperature (above $2000^{\circ} \mathrm{C}$ ) or large surface tension ${ }^{28-30}$. Therefore from a completely different approach based on composite fluid delivery mechanism (Fig. 1), this study successfully developed an entirely-automatic and cost effective way for printing the liquid metal inks.

Through introducing the tapping mode enabled composite fluid transport mechanism to reliably deliver and transfer electronics materials, an extremely easy going automatic printing of the liquid metal ink to quickly print out various high precision electronic patterns was established. Specifically, a printing head pre-loaded with liquid EGaIn 24.5 alloy was developed. Combining the high density, favorable fluidity, driving capability of rolling bead and excellent wettability of the liquid metal ink on matched substrate, the present printing head is capable of fluently drawing conductive structures on the substrate. Further, to realize a straight forward way for electronics making just like clicking a mouse to print the required photos via printers in the office, a notebook computer controlled plotting platform with innovative printing head, ink and software was set up for the automatic printing of the liquid metal. As a result, a diverse range of complex electrically conductive patterns were directly manufactured, in line with the selected drafts stored in the database. At this stage, the total cost for making the machine prototype already reached very low price. This guarantees tremendous potential of the technology for a large scale personal use in the coming time.

1. Sirringhaus, H. et al. High-resolution inkjet printing of all-polymer transistor circuits. Science 290, 2123-2126 (2000).

2. Yan, H. et al. A high-mobility electron-transporting polymer for printed transistors. Nature 457, 679-686 (2009).

3. Nomura, K. et al. Room-temperature fabrication of transparent flexible thin-film transistors using amorphous oxide semiconductors. Nature 432, 488-492 (2004).

4. Park, S. I. et al. Printed assemblies of inorganic light-emitting diodes for deformable and semitransparent displays. Science 325, 977-981 (2009).

5. Ahn, B. Y. et al. Omnidirectional printing of flexible, stretchable, and spanning silver microelectrodes. Science 323, 1590-1593 (2009).

6. Angmo, D., Sweelssen, J., Andriessen, R., Galagan, Y. \& Krebs, F. C. Inkjet printing of back electrodes for inverted polymer solar cells. Adv. Energy Mater. 3, 1230-1237 (2013).

7. McAlpine, M. C., Ahmad, H., Wang, D. \& Heath, J. R. Highly ordered nanowire arrays on plastic substrates for ultrasensitive flexible chemical sensors. Nature Mater. 6, 379-384 (2007)

8. Adams, J. J. et al. Conformal printing of electrically small antennas on threedimensional surfaces. Adv. Mater. 23, 1335-1340 (2011).

9. Marinov, V. R. et al. Direct-write vapor sensors on FR4 plastic substrates. IEEE Sens. J. 7, 937-944 (2007)

10. Cho, J. et al. Efficiency enhanced emitter wrap-through (EWT) screen-printed solar cells with non-uniform thickness of silicon nitride passivation layer in viaholes. Sol. Energy 90, 188-194 (2013).

11. Forrest, S. R. The path to ubiquitous and low-cost organic electronic appliances on plastic. Nature 428, 911-918 (2004).

12. Fukuda, K. et al. Strain sensitivity and durability in p-type and n-type organic thin-film transistors with printed silver electrodes. Sci. Rep. 3, 2048 (2013).

13. Hösel, M. \& Krebs, F. C. Large-scale roll-to-roll photonic sintering of flexo printed silver nanoparticle electrodes. J. Mater. Chem. 22, 15683-15688 (2012). 
14. Cao, Q. et al. Medium-scale carbon nanotube thin-film integrated circuits on flexible plastic substrates. Nature 454, 495-500 (2008).

15. LeMieux, M. C. et al. Self-Sorted, aligned nanotube networks for thin-film transistors. Science 321, 101-104 (2008).

16. Shi, J., Guo, C. X., Chan-Park, M. B. \& Ming, C. All-printed carbon nanotube fin FETs on plastic substrates for high-performance flexible electronics. Adv. Mater. 24, 358-361 (2012).

17. Sun, Y. \& Rogers, J. A. Inorganic semiconductors for flexible electronics. $A d v$. Mater. 19, 1897-1916 (2007).

18. Han, S. T. et al. Microcontact printing: microcontact printing of ultrahigh density gold nanoparticle monolayer for flexible flash memories. Adv. Mater. 24, 3556-3561 (2012).

19. Yue, W., Li, C. W., Xu, T. \& Yang, M. Screen printing of solder resist as master substrates for fabrication of multi-level microfluidic channels and flask-shaped microstructures for cell-based applications. Biosensors and Bioelectronics 41, 675-683 (2013)

20. Bae, S. et al. Roll-to-roll production of 30-inch graphene films for transparent electrodes. Nature Nanotech. 5, 574-578 (2010).

21. Hansen, C. J. et al. Inkjet printing: High-throughput printing via microvascular multinozzle arrays. Adv. Mater. 25, 96-102 (2013).

22. Kim, N. S. \& Han, K. N. Future direction of direct writing. J.Appl.Phys. 108, 102801-1-6 (2010)

23. Gao, Y., Li, H. Y. \& Liu, J. Directly writing resistor, inductor and capacitor to composite functional circuits: a super-simple way for alternative electronics. PLoS ONE 8, e69761 (2013).

24. Jeong, S. H. et al. Liquid alloy printing of microfluidic stretchable electronics. Lab Chip 12, 4657-4664 (2012).

25. Kramer, R. K., Majidi, C. \& Wood, R. J. Masked deposition of gallium-indium alloys for liquid-embedded elastomer conductors. Adv. Func. Mater. 23, 5292-5296 (2013).

26. Tabatabai, A., Fassler, A., Usiak, C. \& Majidi, C. Liquid-phase gallium-indium alloy electronics with microcontact printing. Langmuir 29, 6194-6200 (2013).

27. Ladd, C., So, J. H., Muth, J. \& Dickey, M. D. 3D printing of free standing liquid metal microstructures. Adv. Mater. 25, 5081-5085 (2013).

28. Morley, N. B., Burris, J., Cadwallader, L. C. \& Nornberg, M. D. GaInSn usage in the research laboratory. Rev. Sci. Instrum. 79, 056107-056107-3 (2008).

29. Gao, Y., Li, H. Y. \& Liu, J. Direct writing of flexible electronics through room temperature liquid metal ink. PLoS ONE 7, e45485 (2012).
30. Zheng, Y., He, Z. Z., Gao, Y. \& Liu, J. Direct desktop printed-circuits-on-paper flexible electronics. Sci. Rep. 3, 1786-1-7 (2013).

31. Tobjörk, D. \& Österbacka, R. Paper electronics. Adv. Mater. 23, 1935-1961 (2011).

32. Zheng, Y., Zhang, Q. \& Liu, J. Pervasive liquid metal based direct writing electronics with roller-ball pen. AIP Adv. 3, 112117-1-6 (2013).

\section{Acknowledgments}

We would like to thank B.W. Chen for machine development and X. Xue for computational grid generation.

\section{Author contributions}

Y.Z. performed the experiments, analyzed the data and wrote the manuscript; Z.Z.H. performed the theoretical simulation, analyzed the mechanism and wrote the manuscript; J.Y. performed part of the experiments, analyzed the data, and wrote the manuscript; J.L. conceived the project, designed the work, performed part of the experiments, and wrote the manuscript. All authors discussed the results and commented on the manuscript.

\section{Additional information}

Supplementary information accompanies this paper at http://www.nature.com/ scientificreports

Competing financial interests: The authors declare no competing financial interests

How to cite this article: Zheng, Y., He, Z.-Z., Yang, J. \& Liu, J. Personal electronics printing via tapping mode composite liquid metal ink delivery and adhesion mechanism. Sci. Rep. 4, 4588; DOI:10.1038/srep04588 (2014)

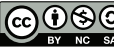

This work is licensed under a Creative Commons Attribution-NonCommercialShareAlike 3.0 Unported License. The images in this article are included in the article's Creative Commons license, unless indicated otherwise in the image credit; if the image is not included under the Creative Commons license, users will need to obtain permission from the license holder in order to reproduce the image. To view a copy of this license, visit http://creativecommons.org/licenses/by-nc-sa/3.0/ 